\title{
Recurrent Acute Myeloid Leukemia
}

National Cancer Institute

\section{Source}

National Cancer Institute. Recurrent Acute Myeloid Leukemia. NCI Thesaurus. Code C148427.

The reemergence of acute myeloid leukemia after a period of remission. 